\title{
A non-reflexive Banach space with all contractions mean ergodic
}

\author{
Vladimir P. Fonf, Michael Lin \\ Ben-Gurion University \\ Przemyslaw Wojtaszczyk \\ University of Warsaw
}

May 4, 2009

Dedicated to the memory of Aryeh Dvoretzky

\begin{abstract}
We construct on any quasi-reflexive of order 1 separable real Banach space an equivalent norm, such that all contractions on the space and all contractions on its dual are mean ergodic, thus answering negatively a question of Louis Sucheston.
\end{abstract}

\section{Introduction}

A linear operator $T$ on a (real or complex) Banach space $X$ is called mean ergodic if the limit

$$
E(T) x:=\lim _{n \rightarrow \infty} \frac{1}{n} \sum_{k=1}^{n} T^{k} x \quad \text { exists } \quad \forall x \in X .
$$

Mean ergodicity was shown by Von-Neumann (1931) for unitary operators on Hilbert spaces, by Riesz (1937) for contractions on Hilbert spaces, and (independently) by Lorch (1939), Kakutani (1938) and Yosida (1938) for power-bounded operators on reflexive spaces. We refer the reader to [9] for proofs, discussion and references. A natural question is whether reflexivity is necessary. In [6] the authors proved that if every power-bounded operator on a Banach space with a basis is mean ergodic, then the space must be reflexive; it is still not known if the same holds without the existence of a basis. For additional references related to this question see $[6]$.

Sucheston [18] posed the following question: If every contraction in a Banach space is mean ergodic, must the space be reflexive? In this paper we construct an example which gives a negative answer to Sucheston's question. 


\section{Operators on quasi-reflexive spaces of order 1}

Definition A Banach space $X$ is quasi-reflexive of order 1 if $\operatorname{dim} X^{* *} / X=1$, where we always consider $X \subset X^{* *}$ via the canonical isometric embedding. The first example of such a space (over $\mathbb{R}$, separable with a basis), and one of the important ones, is the James space [7] (for more of its properties see [4]).

Throughout this note we consider Banach spaces over $\mathbb{R}$.

For the sake of completeness we include the following result of $[13, \S 2.2]$.

Proposition 1. Let $X$ be quasi-reflexive of order 1. Then there exists a linear, multiplicative functional $q: L(X) \rightarrow \mathbb{R}$ of norm 1 such that $\operatorname{ker} q$ is exactly the space of weakly compact operators from $X$ into $X$.

Proof. For $T: X \rightarrow X$ let us consider $T^{* *}: X^{* *} \rightarrow X^{* *}$. Since $T^{* *}(X) \subset X$ we see that it induces an operator $\widetilde{T^{* *}}: X^{* *} / X \rightarrow X^{* *} / X$. It is easy to check that $\left\|\widetilde{T^{* *}}\right\| \leq\left\|T^{* *}\right\|=\|T\|$. Since $X^{* *} / X$ is one-dimensional, $\widetilde{T^{* *}}$ is a multiplication by a number which we denote by $q(T)$. It is clear that the map $q$ is the desired norm 1 linear multiplicative functional. The kernel of $q$ consists of those operators $T: X \rightarrow X$ such that $T^{* *}\left(X^{* *}\right) \subset X$, but this is exactly the set of all weakly compact operators.

Now for $T: X \rightarrow X$ we write $T=q(T) I+(T-q(T) I)$. Since $q(T-q(T) I)=$ 0 , by the proposition $W:=T-q(T) I$ is weakly compact.

If $T: X \rightarrow X$ is power-bounded we get

$$
c \geq\left\|T^{n}\right\| \geq\left|q\left(T^{n}\right)\right|=|q(T)|^{n}
$$

for $n=1,2, \ldots$ so $|q(T)| \leq 1$. Let us summarize this discussion with

Corollary 1. Every linear operator $T$ on $X$ quasi-reflexive of order 1 has a unique decomposition as $T=\lambda I+W$ where $\lambda \in \mathbb{R}$ and $W$ is weakly compact. If $T$ is power-bounded, then $|\lambda| \leq 1$.

Proposition 2. Let $X$ be quasi-reflexive of order 1. Suppose $T=\lambda I+W$ : $X \rightarrow X$ is power-bounded, with $\lambda \neq 1$. Then both $T$ and $T^{*}$ are mean-ergodic.

Proof. For the space of fixed points of $T$, denoted by Fix $(T)$, we have that

$$
\text { Fix }(T)=\{x \in X: \lambda x+W(x)=x\}=\{x \in X: W(x)=(1-\lambda) x\}
$$

is an eigenspace corresponding to a non-zero eigenvalue of a weakly compact operator, so it is a reflexive space.

Analogous arguments show that

$$
\operatorname{Fix}\left(T^{* *}\right)=\left\{x^{* *} \in X^{* *}: W^{* *}\left(x^{* *}\right)=(1-\lambda) x^{* *}\right\} .
$$

Since $W$ is weakly compact, we have $W^{* *}\left(X^{* *}\right) \subset X$, which implies Fix $\left(T^{* *}\right)=$ Fix $(T)$. It is known that Fix $\left(T^{*}\right)$ always separates Fix $(T)$ (e.g. use [9, Theorem 2.1 .3 , p. 73]), so in our case we get that Fix $\left(T^{*}\right)$ separates Fix $\left(T^{* *}\right)$. By Sine's criterion [17], [9, p. 74], we obtain that $T^{*}$ is mean-ergodic. But analogously Fix $\left(T^{* *}\right)$ always separates Fix $\left(T^{*}\right)$, so in our case Fix $(T)$ separates Fix $\left(T^{*}\right)$, and by Sine's criterion $T$ is mean-ergodic. 
Remark. It was shown in [6, Theorem 5] that for $T$ power-bounded on a quasi-reflexive space of order 1 we always have $T$ or $T^{*}$ mean ergodic, and if the space has a basis there exists $T$ power-bounded which is not mean ergodic; by the previous proposition this $T$ is of the form $I+W$ with $W$ weakly compact.

\section{Renorming spaces with separable second dual}

In this section $X$ is a Banach space over $\mathbb{R}$. We use the standard notations

$S_{X}=S_{(X,\|\cdot\|)}=\{x \in X:\|x\|=1 \|\} \quad$ and $\quad B_{X}=B_{(X,\|\cdot\|)}=\{x \in X:\|x\| \leq 1\}$

for the unit sphere and the closed unit ball of $X$, respectively.

Proposition 3. Let $X$ be a non-reflexive Banach space with $X^{* *}$ separable. Then there exist an equivalent norm $\left\||\cdot \||\right.$ on $X$, a functional $f_{0} \in S_{\left(X^{* *},\||\cdot \||)\right.} \backslash X$, and a functional $F_{0} \in S_{\left(X^{* * *},\||\cdot \||)\right.} \cap X^{\perp}$ such that

(i) $F_{0}\left(f_{0}\right)=1$ and $F_{0}(g)<1$ for any $g \in B_{\left(X^{* *},\|\cdot \cdot\| \mid\right)}, g \neq f_{0}$.

(ii) If $H \in S_{\left(X^{* * *},\|\cdot\| \cdot \|\right)}$ and $H\left(f_{0}\right)=1$,

then $H \in X^{\perp}$. Moreover, if there exists a Banach space $Y$ such that $X=Y^{*}$ (isometrically), then the norm $\||\cdot \||$ can be taken as a dual norm.

Proof. We prove first the case that $X=Y^{*}$; we will then indicate how to modify the proof for the non-dual case.

As usual we assume that $Y \subset Y^{* *}=X^{*}$ and $X=Y^{*} \subset Y^{* * *}=X^{* *}$, under the canonical isometric embeddings.

Claim 1. For any $y^{\perp} \in S_{Y^{\perp}} \subset Y^{* * *}$, we have $d\left(y^{\perp}, Y^{*}\right) \geq 1 / 2$. Proof of Claim. For $y^{*} \in Y^{*}$ we have $\left\|y^{\perp}-y^{*}\right\| \geq \sup _{\|y\| \leq 1}\left|\left(y^{\perp}-y^{*}\right)(y)\right|=\left\|y^{*}\right\|$. Hence $\left\|y^{\perp}-y^{*}\right\| \geq \max \left\{\left\|y^{\perp}\right\|-\left\|y^{*}\right\|,\left\|y^{*}\right\|\right\} \geq \frac{1}{2}\left\|y^{\perp}\right\|=\frac{1}{2}$, which proves the claim.

We construct a new norm $\left\||\cdot \||\right.$ in 2 steps. Pick a functional $f \in S_{Y^{\perp}}$. Clearly, $f \in S_{X^{* *}} \backslash X$, and by Claim $1 d(f, X) \geq 1 / 2$. By the Hahn-Banach theorem there is $F \in S_{X^{* * *}} \cap X^{\perp}$ such that $F(f)=d(f, X) \geq 1 / 2$. Next take a sequence $\left\{x_{n}\right\} \subset S_{X}$ such that $w^{*}-\lim x_{n}=f$ (in the $w^{*}$-topology of $X^{* *}$ ). Put

$$
W=\operatorname{cl} \operatorname{co}\left\{B_{X} \cup\left\{ \pm 3 x_{n}\right\}_{n=1}^{\infty}\right\}, \quad W^{* *}=w^{*}-\operatorname{cl} W .
$$

Since $f \in Y^{\perp}$ it follows that $w^{*}-\lim x_{n}=0$ in $w^{*}$-topology defined on $X$ by its predual $Y$. Since $B_{X}$ is $w^{*}$-compact (in the $w^{*}$-topology defined on $X$ by the predual $Y$ ), it easily follows that $W$ is $w^{*}$-compact (indeed, since $w^{*}-\lim x_{n}=0$, it follows that $A=\operatorname{cl} \operatorname{co}\left\{ \pm 3 x_{n}\right\}$ is $w^{*}$-closed, and hence $W=\operatorname{co}\left\{A \cup B_{X}\right\}$ is $w^{*}$-compact, by [3, Lemma V.2.5]). By Milman's theorem [12] (see [16, Prop. $1.5])$,

$$
\operatorname{ext} W^{* *} \subset \operatorname{ext} B_{X^{* *}} \cup\left\{ \pm 3 x_{n}\right\}_{n=1}^{\infty} \cup\{ \pm 3 f\} .
$$

Since $X^{* *}$ is separable, $W^{* *}$ is a weak-* compact convex set with ext $W^{* *}$ separable, so by [8],[5] (see also [16, p. 26]), we have that $W^{* *}=\operatorname{cl} \operatorname{co}\left\{\operatorname{ext} W^{* *}\right\}-$ the norm closed convex hull (this can be deduced also from the Bessaga-Pełczyński theorem [2]). It follows that

$$
\sup F\left(W^{* *}\right)=\sup F\left(\operatorname{ext} W^{* *}\right) .
$$


Since $\|F\|=1$ and $F \in X^{\perp}$, by (1) we have $\sup F\left(W^{* *}\right)=F(3 f)>1$, and, moreover, $F(g)<F(3 f)$ for any $g \in W^{* *}, g \neq 3 f$.

Let $\left\{u_{i}\right\}_{i=1}^{\infty} \subset B_{X}$ be a sequence dense in $B_{X}$. Put

$$
\begin{gathered}
f_{0}=3 f, \quad t_{n}=3 x_{n}, \quad F_{0}=\frac{1}{F(3 f)} F, \quad K=\operatorname{clco}\left\{ \pm 2^{-i} u_{i}\right\}_{i=1}^{\infty}, \\
T_{n}=\left\{\lambda t_{n}+x: x \in K, \lambda^{2}+\|x\|^{2} \leq 1\right\}, \quad n=1,2, \ldots, \\
T_{0}=\left\{\lambda f_{0}+x: x \in K, \lambda^{2}+\|x\|^{2} \leq 1\right\},
\end{gathered}
$$

Since $K$ is symmetric, each $T_{n}$ is symmetric, and we define

$$
V=\operatorname{cl} \operatorname{co}\left\{W \cup \cup_{n=1}^{\infty} T_{n}\right\}, \quad V^{* *}=w^{*}-\mathrm{cl} V .
$$

Claim 2. $V$ is $w^{*}$-closed in the $w^{*}$-topology on $X$ defined by the predual $Y$. Proof of Claim. Put $A=w^{*}-\operatorname{clco} \cup_{n=1}^{\infty} T_{n}$. An easy consideration shows that $w^{*}-\operatorname{cl} \cup_{n=1}^{\infty} T_{n}=\cup_{n=1}^{\infty} T_{n} \cup K$ (recall that $\left.w^{*}-\lim t_{n}=0\right)$. Since $A$ is a weak-* compact convex set (in $X=Y^{*}$ ), by Milman's theorem $\operatorname{ext} A \subset$ $w^{*}-\operatorname{cl} \cup_{n=1}^{\infty} T_{n}=\cup_{n=1}^{\infty} T_{n} \cup K$, so by [8],[5] $A=\operatorname{cl} \operatorname{co}\left\{\cup_{n=1}^{\infty} T_{n} \cup K\right\}$. Since $K \subset B_{X} \subset W$, it follows that $A \subset V$. Finally, since also $W$ is $w^{*}$-compact and convex, we obtain (using [3, Lemma V.2.5])

$$
V \subset w^{*}-\operatorname{cl} \operatorname{co}\{W \cup A\}=\operatorname{co}\{W \cup A\} \subset V,
$$

hence $V=w^{*}-\operatorname{cl} \operatorname{co}\{W \cup A\}$ is $w^{*}$-closed, which finishes the proof of Claim 2 .

Since $V$ is a bounded closed convex symmetric subset of $X$ containing $B_{X}$, it is easy to show that $\|\mid x\|:=\inf \left\{t>0: \frac{1}{t} x \in V\right\}$ defines an equivalent norm on $X$ with $B_{(X,\|\cdot \cdot\|)}=V$. From Claim 2 we get that $\||\cdot|\| \mid$ is a dual norm (see, e.g., [19]). Next, by Milman's theorem (in the weak-* topology of $X^{* *}$ )

$$
\operatorname{ext} V^{* *} \subset W^{* *} \cup \cup_{n=0}^{\infty} T_{n},
$$

and since $V^{* *}$ is weak-* compact convex, it is the norm-closed convex hull of $\operatorname{ext} V^{* *}([8],[5])$, and by the choice of $F_{0}$ (in particular, $F_{0} \in X^{\perp}$ ), we have

$$
\sup F_{0}\left(V^{* *}\right)=\sup F_{0}\left(\operatorname{ext} V^{* *}\right)=\sup F_{0}\left(W^{* *}\right)=F_{0}\left(f_{0}\right)=1 .
$$

Moreover, $f_{0}$ is the only point in $W^{* *} \cup \cup_{n=0}^{\infty} T_{n}$ where $F_{0}$ attains the value 1 . Indeed, for $W^{* *}$ it was already mentioned above, while for the set $\cup_{n=0}^{\infty} T_{n}$ it easily follows from $F_{0} \in X^{\perp}$. Finally assume that $H \in X^{* * *}$ satisfies $H\left(f_{0}\right)=$ $1=\max H\left(V^{* *}\right)$; we prove that $H \in X^{\perp}$. Fix $y \in K$ and put

$$
D=\left\{a f_{0}+b y: a^{2}+b^{2} \leq 1\right\} .
$$

We show that $D \subset T_{0}$ : For $a f_{0}+b y \in D$ we have $a^{2}+b^{2} \leq 1$. Since $K \subset B_{X}$ is absolutely convex, $y \in K$ and $|b| \leq 1$ imply that $x=b y \in K$, and $\|y\| \leq 1$ yields $a^{2}+\|x\|^{2} \leq a^{2}+b^{2} \leq 1$. Therefore $a f_{0}+b y \in T_{0} \subset V^{* *}$. Thus we have

$$
1=H\left(f_{0}\right)=\max H(D) .
$$


Let $H(y)=\gamma$. We then have $\left(\sqrt{1+\gamma^{2}}\right)^{-1}\left(f_{0}+\gamma y\right) \in D$ and

$$
H\left(\frac{1}{\sqrt{1+\gamma^{2}}} f_{0}+\frac{\gamma}{\sqrt{1+\gamma^{2}}} y\right)=\frac{1}{\sqrt{1+\gamma^{2}}}+\frac{\gamma^{2}}{\sqrt{1+\gamma^{2}}}=\sqrt{1+\gamma^{2}} \leq 1
$$

so we must have $H(y)=0$. Since $y \in K$ is arbitrary and $\operatorname{cl} \operatorname{span} K=X$, it follows that $H \in X^{\perp}$.

When $X$ is not a dual space, we skip Claim 1 and start directly by taking $f \in S_{X^{* *}} \backslash X$ with $d(f, X)>1 / 3$, and then procced with the same proof, ignoring (the now unnecessary) Claim 2.

Remark. Clearly, the condition $X^{* *}$ is separable may be weakened. For instance, the same proof works, using deeper results, if $X^{*}$ is separable and does not contain $\ell_{1}$ (use the remark at the end of [15], instead of [8],[5] or [2], for expressing $W^{* *}$ and $V^{* *}$ as the norm-closed convex hull of their respective extreme points).

Corollary 2. Let $X$ be a separable quasi-reflexive of order 1 Banach space. Then there exist an equivalent norm $\|\cdot \cdot\| \mid$ on $X$, a functional $f_{0} \in S_{\left(X^{* *},\|\cdot\| l\right)} \backslash X$, and a functional $F_{0} \in S_{\left(X^{* * *},\|\cdot \cdot\| \mid\right)} \cap X^{\perp}$, such that

(i) $F_{0}\left(f_{0}\right)=1$ and $F_{0}(g)<1$ for any $g \in B_{\left(X^{* *},\|\mid \cdot\| l\right),} g \neq f_{0}$.

(ii) $F_{0}$ is the only functional in $S_{\left(X^{* * *},\|\cdot \cdot\| \mid\right)}$ with $F_{0}\left(f_{0}\right)=1$. Moreover, if there exists a Banach space $Y$ such that $X=Y^{*}$ (isometrically), then the norm $\||\cdot| \mid$ can be taken as a dual norm.

Proof. For part (ii), note that $X^{* *}=X \oplus\left[f_{0}\right]$ by quasi-reflexivity of order 1 .

\section{Mean ergodicity of contractions}

In this section we construct non-reflexive separable Banach spaces such that every contraction is mean ergodic (abbreviated ME in the sequel).

We start with a general lemma on mean ergodicity.

Lemma 1. Let $R$ be a mean ergodic power-bounded operator on a Banach space $Y$, with ergodic projection $E y=\lim \frac{1}{n} \sum_{k=1}^{n} R^{k} y$. Then $E^{*}\left(Y^{*}\right)=$ Fix $\left(R^{*}\right)$.

Proof. Since $R E=E R=E=E^{2}$, we have $R^{*} E^{*}=E^{*}$, which yields $E^{*}\left(Y^{*}\right) \subset$ Fix $\left(R^{*}\right)$. For the converse, let $y^{*} \in \operatorname{Fix}\left(R^{*}\right)$. Then for $y \in Y$ we have

$$
E^{*} y^{*}(y)=y^{*}(E y)=y^{*}\left(\lim _{n} \frac{1}{n} \sum_{k=1}^{n} R^{k} y\right)=y^{*}(y) .
$$

Throughout this section, we assume that $X$ is a separable Banach space quasi-reflexive of order 1 , endowed with the norm given by Corollary 2 , and we use the notations of Corollary 2. We will show that every contraction on $X$ is mean ergodic. 
Lemma 2. Let $Q$ be a norm 1 projection from $\left(X^{* *},\||\cdot \||)\right.$ onto the onedimensional subspace spanned by $f_{0}$. Then $Q x^{* *}=F_{0}\left(x^{* *}\right) f_{0}$.

Proof. Clearly $Q x^{* *}=\alpha\left(x^{* *}\right) f_{0}$, with $\alpha$ linear (by linearity of $Q$ ), and $\||\alpha \||=1$ since $\left|\alpha\left(x^{* *}\right)\right|=\left\|\left|Q x^{* *}\right|\right\|\left|\leq\left\|x^{* *}\right\|\right|$ with equality on $Q\left(X^{* *}\right) \neq\{0\}$. Now

$$
\alpha\left(f_{0}\right) f_{0}=Q f_{0}=Q^{2} f_{0}=\alpha^{2}\left(f_{0}\right) f_{0}
$$

so $\alpha\left(f_{0}\right)=1$ since $Q \neq 0$. By Corollary $2(\mathrm{ii}), \alpha=F_{0}$.

Lemma 3. Let $T=I+W$ be a contraction in $(X,\||\cdot \||)$ with $W$ weakly compact. Then $f_{0} \in$ Fix $\left(T^{* *}\right)$.

Proof. Since $W$ is weakly compact, it follows that $W^{* *} f_{0} \in X$, and hence $F_{0}\left(W^{* *} f_{0}\right)=0$. Hence

$$
1 \geq\left\|\left|T ^ { * * } f _ { 0 } \left\|\left|=\left\|\left|f_{0}+W^{* *} f_{0} \|\right| \geq F_{0}\left(f_{0}\right)+F_{0}\left(W^{* *} f_{0}\right)=1+F_{0}\left(W^{* *} f_{0}\right)=1 .\right.\right.\right.\right.\right.
$$

Therefore $\left\|\mid f_{0}+W^{* *} f_{0}\right\| \|=1$, and we conclude that $F_{0}$ attains its norm on $f_{0}+W^{* *} f_{0}$. Corollary $2(\mathrm{i})$ yields that $W^{* *} f_{0}=0$, i.e. $f_{0} \in \operatorname{Fix}\left(T^{* *}\right)$.

Theorem 1. Every separable Banach space which is quasi-reflexive of order 1 has an equivalent norm in which any contraction is mean ergodic.

Proof. Endow $X$ with the norm $\||\cdot \||$ obtained in Corollary 2. Let $T: X \rightarrow X$ be a contraction. By Proposition 2 we have to prove only the case $T=I+W$ where $W$ is weakly compact (to which Lemma 3 is applicable). By [6], $T$ or $T^{*}$ (or both) is ME, so without loss of generality we may assume that $T^{*}$ is ME. Besides, we can assume that Fix $\left(T^{*}\right) \neq\{0\}$ (otherwise $X=\overline{(I-T) X}$ so $T$ is $\mathrm{ME}$ and we are done).

Define

$$
P x^{*}=\lim \frac{1}{n} \sum_{i=1}^{n} T^{* k} x^{*}, \quad x^{*} \in X^{*} .
$$

Clearly, $P$ is a projection onto Fix $\left(T^{*}\right)$, and since Fix $\left(T^{*}\right) \neq\{0\}$ and $\||T \|| \leq 1$, it follows that $\left\||P \||=1\right.$ (i.e. $P$ is not 0 ). Since $T^{*}$ is $\mathrm{ME}$ we have the following ergodic decomposition

$$
X^{*}=P\left(X^{*}\right) \oplus \overline{\left(I-T^{*}\right) X^{*}}=F\left(T^{*}\right) \oplus \overline{W^{*} X^{*}} .
$$

By Lemma $3 f_{0} \in$ Fix $\left(T^{* *}\right)$, so Fix $\left(T^{* *}\right)=$ Fix $(T) \oplus\left[f_{0}\right]$. Define

$$
Q x^{* *}=F_{0}\left(P^{*} x^{* *}\right) f_{0} .
$$

Then $Q f_{0}=f_{0}$ and $\|\mid Q\|=1, Q^{2}=Q$. Lemma 2 yields that $Q x^{* *}=$ $F_{0}\left(x^{* *}\right) f_{0}$. Hence $\operatorname{Ker} Q=\operatorname{Ker} F_{0}=X$ (we use here that $X$ is quasi-reflexive of order 1). Therefore $\operatorname{Ker} P^{*} \subset X$. By Lemma 1 Fix $\left(T^{* *}\right)=P^{*}\left(X^{* *}\right)$, and

$$
X^{* *}=P^{*} X^{* *} \oplus \operatorname{Ker} P^{*}=\operatorname{Fix}\left(T^{* *}\right) \oplus \operatorname{Ker} P^{*}=\left[f_{0}\right] \oplus \operatorname{Fix}(T) \oplus \operatorname{ker} P^{*},
$$

and hence

$$
X=\operatorname{Fix}(T) \oplus \operatorname{Ker} P^{*}
$$


Mean ergodicity of $T^{*}$ implies

$$
P^{*} x^{* *}=w^{*}-\lim \frac{1}{n} \sum_{k=1}^{n} T^{* * k} x^{* *} .
$$

If $P^{*} x^{* *}=0$ then $x^{* *}=x \in X$, and we have $\frac{1}{n} \sum_{k=1}^{n} T^{k} x \rightarrow 0$ weakly, hence in norm (e.g. [9, p. 72]). The decomposition (2) yields that $T$ is ME.

Theorem 2. Let $X$ be quasi-reflexive of order 1 , with $\||\cdot|||$ the norm defined by Corollary 2, and let $T$ be a contraction on $\left(X,\||\cdot \||)\right.$; then $T^{*}$ is mean ergodic.

Proof. By Proposition 2 it remains to prove the theorem only for $T=I+W$ with $W$ weakly compact, which we now assume. From Theorem 1 we know that $T$ is mean ergodic, and denote $E x:=\lim _{n \rightarrow \infty} \frac{1}{n} \sum_{k=1}^{n} T^{k} x$. Then $E$ is a projection onto Fix $(T)$, and $E^{2}=E=E T=T E$. Hence by Lemma $1 E^{*}$ is a projection of $X^{*}$ onto Fix $\left(T^{*}\right)$, and $E^{* *}$ projects $X^{* *}$ into Fix $\left(T^{* *}\right)$, so we have $E^{* *} f_{0} \in \operatorname{Fix}\left(T^{* *}\right)$. By Lemma $3 f_{0} \in \operatorname{Fix}\left(T^{* *}\right)$, and the decomposition $X^{* *}=X \oplus\left[f_{0}\right]$ yields that $T^{* *}$ is mean ergodic. The ergodic decomposition of $X$ by mean ergodicity of $T$ yields

$$
X^{* *}=X \oplus\left[f_{0}\right]=\operatorname{Fix}(T) \oplus \overline{(I-T) X} \oplus\left[f_{0}\right]
$$

so Fix $\left(T^{* *}\right)=\operatorname{Fix}(T) \oplus\left[f_{0}\right]$. Hence $E^{* *} f_{0}=\lambda f_{0}+y_{0}$ with $y_{0} \in \operatorname{Fix}(T)$. Since $E^{* *} \mid X=E$, we have $E^{* *} y_{0}=y_{0} \in X$. The functional $F_{0}$ is in $X^{\perp}$, so we have $\lambda=F_{0}\left(\lambda f_{0}+y_{0}\right)=F_{0}\left(E^{* *} f_{0}\right)=F_{0}\left(E^{* *} E^{* *} f_{0}\right)=F_{0}\left(\lambda^{2} f_{0}+\lambda y_{0}+E^{* *} y_{0}\right)=\lambda^{2}$.

Case $(i): \lambda=1$. In this case $F_{0}\left(E^{* *} f_{0}\right)=1$, and since $\left\|\left|f_{0} \|\right|=1\right.$ and $\left\||E \|| \leq 1\right.$, Corollary 2 (i) yields $E^{* *} f_{0}=f_{0}$. We have observed that $T^{* *}$ is mean ergodic with the decomposition (3). Since $E^{* *} \mid X=E$ and $E^{* *} f_{0}=f_{0}$, we obtain that $\lim _{n \rightarrow \infty} \frac{1}{n} \sum_{k=1}^{n} T^{* * k} x^{* *}=E^{* *} x^{* *}$. Hence for $x^{*} \in X^{*}$ we have that for every $x^{* *} \in X^{* *}$

$$
x^{* *}\left(\frac{1}{n} \sum_{k=1}^{n} T^{* k} x^{*}\right)=\left(\frac{1}{n} \sum_{k=1}^{n} T^{* * k} x^{* *}\right)\left(x^{*}\right) \rightarrow E^{* *} x^{* *}\left(x^{*}\right)=x^{* *}\left(E^{*} x^{*}\right) .
$$

Thus $\frac{1}{n} \sum_{k=1}^{n} T^{* k} x^{*}$ converges weakly to $E^{*} x^{*}$, and therefore in norm [9, p. 72]. Hence $T^{*}$ is mean ergodic.

Case (ii): $\lambda=0$. In this case we have $E^{* *} f_{0} \in \operatorname{Fix}(T)$, so $E^{* *}\left(\operatorname{Fix}\left(T^{* *}\right)\right) \subset$ Fix $(T)$ (and equality holds). Let $y^{* *} \in \mathrm{Fix}\left(T^{* *}\right)$, and put $y=E^{* *} y^{* *} \in$ Fix $(T)$. Since (for any contraction on a Banach space) Fix $\left(T^{*}\right)$ separates Fix $(T)$, there exists $y^{*} \in \operatorname{Fix}\left(T^{*}\right)$ such that $y^{*}(y) \neq 0$. By Lemma $1 E^{*} y^{*}=$ $y^{*}$, which yields

$$
y^{* *}\left(y^{*}\right)=y^{* *}\left(E^{*} y^{*}\right)=E^{* *} y^{* *}\left(y^{*}\right)=y^{*}(y) \neq 0 .
$$

Hence Fix $\left(T^{*}\right)$ separates Fix $\left(T^{* *}\right)$, so by Sine's criterion $T^{*}$ is mean ergodic.

Lemma 4. Let $X$ be a Banach space such that all contractions are mean ergodic. Then every strongly continuous semi-group of contractions $\left\{T_{t}\right\}_{t>0}$ on $X$ is mean ergodic, i.e.

$$
\lim _{t \rightarrow \infty} \frac{1}{t} \int_{0}^{t} T_{s} x d s \quad \text { exists } \quad \forall x \in X
$$


Proof. For the sake of completeness we indicate the standard proof. For $x \in X$ put $y=\int_{0}^{1} T_{s} x d s$. By the semi-group property $T_{k}=T_{1}^{k}$ and we obtain

$$
\frac{1}{t} \int_{0}^{t} T_{s} x d s=\frac{[t]}{t} \cdot \frac{1}{[t]} \sum_{k=0}^{[t]-1} T_{1}^{k} y+\frac{1}{t} \int_{[t]}^{t} T_{s} x d s \longrightarrow E\left(T_{1}\right) y
$$

by mean ergodicity of the contraction $T_{1}$.

Remark. Mugnolo [14] gave a semi-group analogue of the result of [6].

We can now reinforce our negative answer to Sucheston's question.

Theorem 3. Every separable Banach space $Z$ which is quasi-reflexive of order 1 has an equivalent norm such that all contractions on $Z$ and all contractions on $Z^{*}$ (in the induced dual norm) are mean ergodic. Moreover, every strongly continuous semi-group of contractions on $Z$ or on $Z^{*}$ (in the above norms) is mean ergodic.

Proof. Let $Z$ be a separable Banach space quasi-reflexive of order 1 and let $X=Z^{*}$. Then also $X$ is quasi-reflexive of order 1 (e.g. [4, p. 10]), and by the construction of the norm $\||\cdot \||$ on $X$ obtained in Corollary 2, there is an equivalent norm on $Z$, denoted by $|\cdot|$, for which $\left(X,\||\cdot \||)=(Z,|\cdot|)^{*}\right.$. Let $R$ be a contraction on $(Z,|\cdot|)$. Then $T=R^{*}$ is a contraction on $(X,\||\cdot|\| \mid)$, and by Theorem $2 T^{*}=R^{* *}$ is mean ergodic on $X^{*}=Z^{* *}$. But $R^{* *} \mid Z=R$, so the mean ergodicity of $R^{* *}$ yields mean ergodicity of $R$.

If $T$ is a contraction on $(Z,|\cdot|)^{*}=(X, \||\cdot|||)$, then by Theorem $1 T$ is mean ergodic on $X$.

The mean ergodicity of strongly continuous contraction semi-groups follows from the above and the previous lemma.

Browder [1, Lemma 5] proved that for $T$ power-bounded on a reflexive Banach space $Y$ we have

$$
x \in(I-T) Y \quad \text { if and only if } \quad \sup _{n}\left\|\sum_{k=0}^{n} T^{k} x\right\|<\infty .
$$

Lin and Sine [11] showed that (4) holds also for $Y=L_{1}$ and $T$ any contraction (even not mean ergodic), and gave an example of a mean ergodic contraction $T$ on a subspace of $L_{1}$ for which (4) fails. Thus (4) and mean ergodicity are incomparable. When (4) holds we say that $T$ satisfies Browder's condition.

Lemma 5. Let $T$ be a power-bounded operator on a Banach space $Y$. If $T^{* *}$ is mean ergodic (on $Y^{* *}$ ), then (4) holds.

Proof. Let $\sup _{n}\left\|\sum_{k=0}^{n} T^{k} x\right\|<\infty$. Browder's result was extended in [10] (see also [11]) to show that (4) holds when $Y$ is a dual space and $T$ is a dual operator. We apply this to $T^{* *}$ and obtain that $x \in\left(I-T^{* *}\right) Y^{* *}$. The assumption that $T^{* *}$ is mean ergodic yields, by [11, Theorem 1], that a solution $y^{* *} \in Y^{* *}$ of the equation $\left(I-T^{* *}\right) y^{* *}=x$ is given by

$$
y^{* *}=\lim _{N \rightarrow \infty} \frac{1}{N} \sum_{n=1}^{N} \sum_{k=0}^{n-1} T^{* * k} x=\lim _{N \rightarrow \infty} \frac{1}{N} \sum_{n=1}^{N} \sum_{k=0}^{n-1} T^{k} x,
$$

which shows that $y^{* *} \in Y$ and $x \in(I-T) Y$. 
Theorem 4. Let $Z$ be a separable Banach space which is quasi-reflexive of order 1, endowed with the equivalent norm defined in Theorem 3. Then every contraction on $Z$ and every contraction on $X=Z^{*}$ satisfies Browder's condition.

Proof. Let $T$ be a contraction on $Z$. Then $T^{*}$ is a contraction on $X$, and by Theorem $2 T^{* *}$ is mean ergodic on $X^{*}=Z^{* *}$. Hence $T$ satisfies Browder's condition by the previous lemma.

Now let $T$ be a contraction on $Z^{*}=(X,\|\| \cdot\|\|)$ and let $x \in X$ satisfy $\sup _{n}\left\|\left|\sum_{k=0}^{n} T^{k} x \|\right|<\infty\right.$; then there exists $y^{* *} \in X^{* *}$ with $\left(I-T^{* *}\right) y^{* *}=x$. Recall (Corollary 1 ) that $T=\lambda I+W$ with $W$ weakly compact (and $|\lambda| \leq 1$ ).

Case (i): $\lambda \neq 1$. Since $W^{* *} y^{* *}=z \in X$, we obtain

$$
x=\left(I-T^{* *}\right) y^{* *}=(1-\lambda) y^{* *}-W^{* *} y^{* *}=(1-\lambda) y^{* *}-z,
$$

which yields $y^{* *}=(1-\lambda)^{-1}(x+z) \in X$, so $x \in(I-T) X$.

Case (ii): $\lambda=1$. Let $y^{* *}=y+\alpha f_{0}$, with $y \in X$. By Lemma $3 T^{* *} f_{0}=f_{0}$, so $x=\left(I-T^{* *}\right)\left(y+\alpha f_{0}\right)=(I-T) y$.

\section{Problems}

It was proved in [6] that if $Y$ is a Banach space such that every power-bounded operator defined on any closed subspace of $Y$ is mean ergodic (on that subspace), then $Y$ is reflexive (no basis assumed). The question (related to another question in [18]) is this: If $Y$ is a Banach space such that every contraction defined on any closed subspace is mean ergodic, is $Y$ reflexive?

If the answer to the above is negative, what if both $Y$ and $Y^{*}$ have the above property?

ACKNOWLEDGEMEnTs. The research was started during a visit of the third author to Ben-Gurion University, supported by the Center for Advanced Studies in Mathematics of Ben-Gurion University. The research was completed during a visit of the second author to the Institute of Mathematics of the Polish Academy of Sciences (IMPAN) in Warsaw, supported by the EU project TODEQ. The above authors are grateful for the support and hospitality of their hosts. The third author wishes to thank also the Foundation for Polish Science for its support.

\section{References}

[1] F. Browder, On the iteration of transformations in noncompact minimal dynamical systems, Proc. Amer. Math. Soc. 9 (1958), 773-780.

[2] C. Bessaga and A. Pełczyński, On extreme points in separable conjugate spaces, Israel J. Math. 4 (1966), 262-264.

[3] N. Dunford and J. Schwartz, Linear operators, Part I, Interscience, New York, 1958. 
[4] H. Fetter and B. Gamboa de Buen, The James forest, London Math. Soc. Lecture Notes 236, Cambridge University Press, Cambridge (UK), 1997.

[5] R. Haydon, An extreme point criterion for separability of a dual Banach space, and a new proof of a theorem of Corson, Quarterly J. Math. Oxford 27 (1976), 377-385.

[6] V. Fonf, M. Lin and P. Wojtaszczyk, Ergodic characterizations of reflexivity of Banach spaces, J. Funct. Anal. 187 (2001), 146-162.

[7] R. C. James, Bases and reflexivity of Banach spaces, Ann. Math. 52 (1950), 518-527.

[8] M. I. Kadec (Kadets) and V. P. Fonf, Some properties of the set of extreme points of the unit ball of a Banach space, Mat. Zametki 20 (1976), 315-320 (in Russian; English translation Math. Notes 20 (1976), 737-739).

[9] U. Krengel, Ergodic theorems, De Gruyter, Berlin, 1985.

[10] M. Lin, On quasi-compact Markov operators, Annals Proba. 2 (1974), 464475.

[11] M. Lin and R. Sine, Ergodic theory and the functional equation $(I-T) x=$ y, J. Operator Theory 10 (1983), 153-166.

[12] D.P. Milman, Characteristics of extremal points of regularly convex sets, Doklady Akad. Nauk SSSR 57 (1947), 119-122 (in Russian); MR 9,192 (1948).

[13] B. Mityagin and I. Edelstein, The homotopy type of linear groups of two classes of Banach spaces, Funk. Anal. Pril. 4 (1970), no. 3, 61-72 (in Russian; English translation Funct. Anal. Appl. 4 (1970), 221-231).

[14] D. Mugnolo, A semigroup analogue of the Fonf-Lin-Wojtaszczyk ergodic characterization of reflexive Banach spaces with a basis, Studia Math. 164 (2004), 243-251.

[15] E. Odell and H. P. Rosenthal, A double-dual characterization of separable Banach spaces containing $l^{1}$, Israel J. Math. 20 (1975), 375-384.

[16] R. R. Phelps, Lectures on Choquet's theorem, 2nd ed., Springer Lecture Notes in Math. 1757, Springer, Berlin, 2001.

[17] R. Sine, A mean ergodic theorem, Proc. Amer. Math. Soc. 24 (1970), 438439.

[18] L. Sucheston, Problems, in Probability in Banach spaces, Springer Lecture Notes in Math. 526, pp. 285-290; Springer, Berlin, 1976.

[19] J. P. Williams, A "metric" characterization of reflexivity, Proc. Amer. Math. Soc. 18 (1967), 163-165. 